\title{
Cuando el territorio se hace texto: protagonizando la Tecnicatura Universitaria en Economía Social y Solidaria en la Universidad Nacional de Quilmes
}

\author{
Selva Sena \\ selva_sena@yahoo.com.ar \\ Dalila Sansón
}

dalisanson@yahoo.com.ar

Stella Berón

stella.beron@gamil.com

\section{Daniel García}

dangaroki@gmail.com

Docentes de la Universidad Nacional de Quilmes, Argentina
Integración de la docencia y la extensión /

Intervenciones

RECEPCIÓN: 24/06/16

ACEPTACIÓN FINAL: 10/10/16

\section{Resumen}

La Universidad Nacional de Quilmes creó en Argentina la primera Tecnicatura Universitaria en Economía Social y Solidaria en 2011. Desde 2012, la Tecnicatura se articula con el Diploma de Operador Socioeducativo en Economía Social y Solidaria, oferta curricular de la extensión universitaria, surgida en el marco de vínculos de la Universidad con actores y organizaciones de la Economía Social y Solidaria. Nos proponemos dar a conocer aquí reflexiones y síntesis conceptuales sobre la formación en las Prácticas Profesionalizantes (PP) de la Tecnicatura y su articulación con determinadas experiencias de extensión (por ejemplo: Curso de Extensión). Pretendemos aportar a la comunidad educativa a partir de repensar las prácticas pedagógicas en la Universidad y su responsabilidad social y ofrecer perspectivas analíticas de la complejidad, vínculo con el territorio y fortalecimiento de habilidades subjetivas y colectivas desde una experiencia concreta, con la pretensión de escalar criterios y aspectos generalizables a otras trayectorias de formación similares.

\section{Palabras-clave}

- Prácticas formativas

- Economía Social y Solidaria

- Extensión universitaria

- Territorio

\section{Resumo}

Em 2011, a Universidade Nacional de Quilmes criou na Argentina o primeiro Curso de Técnico Universitário em Economia Social e Solidária. A partir de 2012, o curso se articula com o Diploma de Operador Socioeducativo em Economia Social e Solidária, oferta curricular da extensão universitária, surgida no marco de vínculos da Universidade com atores e organizações da Economia Social e Solidária. Propomo-nos divulgar aqui, reflexões e sínteses conceituais sobre a formação nas Práticas Profesionalizantes deste Curso Técnico e sua articulação com determinadas experiências de extensão (por exemplo: Curso de Extensão). Pretendemos colaborar com a comunidade educativa a partir de repensar as práticas pedagógicas na Universidade e sua responsabilidade social, e oferecer perspectivas analíticas da complexidade, vínculo com o território e fortalecimento de habilidades subjetivas e coletivas, desde uma experiência objetiva, com a intenção de progredir em critérios e aspectos que abrangem outras trajetórias de formação semelhantes.

Palavras-chave

- Práticas formativas

- Economia Social e Solidária

- Extensão universitária

- Território
ción de este artículo Sena, S.; Sansón, D., Berón, S. y García, D. (2016). Cuando el territorio se hace texto: protagonizando la Tecnicatura Universitaria en Economía Social y Solidaria en la Universidad Nacional de Quilmes. En Revista +E versión digital, (6), pp. 430-437. Santa Fe, Argentina: Ediciones UNL. 


\section{Introduciendo una intervención territorial}

En este texto, situando nuestra intervención en el marco de las Prácticas Profesionalizantes de la Tecnicatura en Economía Social y Solidaria de la Universidad Nacional de Quilmes (TUESS), desarrollaremos una serie de precisiones conceptuales y metodológicas sobre la instrumentación de las prácticas formativas en el campo de la Economía Social y Solidaria (ESS) y su abordaje en un proyecto en particular a modo de ejemplo. Como conclusión, encontraremos una serie de aproximaciones analíticas para abordar la curricularización de la extensión en el área de la ESS. La Tecnicatura surge como continuidad del Diploma de Extensión Universitaria de Operador Socioeducativo de la ESS (DOSESS) y constituye una trayectoria universitaria integral de formación en ESS. Se origina a partir de la demanda de formación integral de organizaciones y emprendimientos de la ESS vinculados a la Universidad en proyectos y actividades de extensión y de las políticas públicas en el área, articulando sus necesidades. La ESS, según Coraggio, coloca en el centro aquellas estrategias pedagógicas que exigen, ante cada situación concreta, que sus agentes inventen de manera responsable:

"respuestas y procedimientos en diálogo con los saberes de las personas y grupos a los cuales se dirigen las intervenciones. El trabajo interdisciplinario e interinstitucional y con la participación de los destinatarios de las políticas supone una base de supuestos compartidos, de cuya consolidación es corresponsable la universidad”. (Coraggio, 2015:6)

La Universidad Nacional de Quilmes (UNQ) se ha comprometido de manera corresponsable con el campo de la ESS desde el año 2006 a partir de la generación de una Mesa de Promoción de la ESS desde la Extensión Universitaria en Quilmes, Florencio Varela y Berazategui, con emprendedores, referentes territoriales y organizaciones sociales, en la búsqueda de la obtención de mejores condiciones colectivas para responder a un contexto de creciente desigualdad y precarización social y frente a la recurrencia de crisis económicas (Pastore, 2014).
Según Pastore (2014), esta experiencia de extensión nos ha enfrentado a un escenario poco visibilizado y valorado de actores y agentes con múltiples fragmentaciones y una gran variedad de tensiones y rivalidades políticas e ideológicas, además de una serie de dificultades para aumentar su capacidad de gestión y su sostenibilidad. Lo que desarrollaremos en este texto se enmarca en las propuestas de formación del sector derivadas de ese diagnóstico y esa relación de la Universidad con los actores participantes de los proyectos de extensión de la ESS.

Existen muchas apreciaciones sobre el concepto de la ESS que la refieren a las instituciones, normas y prácticas orientadas a la satisfacción de necesidades y deseos de los sujetos en sus territorios (Coraggio, 2011). Sin embargo, no todas las prácticas populares de satisfacción de necesidades son coincidentes con los principios y prácticas de la ESS en tanto campo socioeconómico, simbólico y político. A nuestro entender, la ESS procura obtener como finalidad social la reproducción ampliada de la vida. Es una economía para la vida, presente y capilarizada en los territorios en prácticas diversas. En función de este objetivo, se analizan y componen los recursos disponibles, en un marco de interacción de los hombres entre sí y con el medio en el que viven. De manera polisémica y multiintegrada, está compuesta por tres dimensiones interrelacionadas (Pastore y Altschuler, 2015): a) La primera alude a las trayectorias empíricas, es decir las formas de organización para hacer economía, en general: asociativas, solidarias, cooperativas, autogestivas y con espíritu democrático. b) La segunda apunta a los proyectos políticos de la sociedad en disputa y a las tensiones inherentes a la adaptación a las lógicas económicas hegemónicas versus la transformación socioeconómica que requiere profundizar la democratización y la solidaridad sistémica.

c) La tercera dimensión aborda los aspectos simbólicos concatenados al desarrollo conceptual y al abordaje cultural, educativo y comunicacional de las prácticas de interacción económica. 


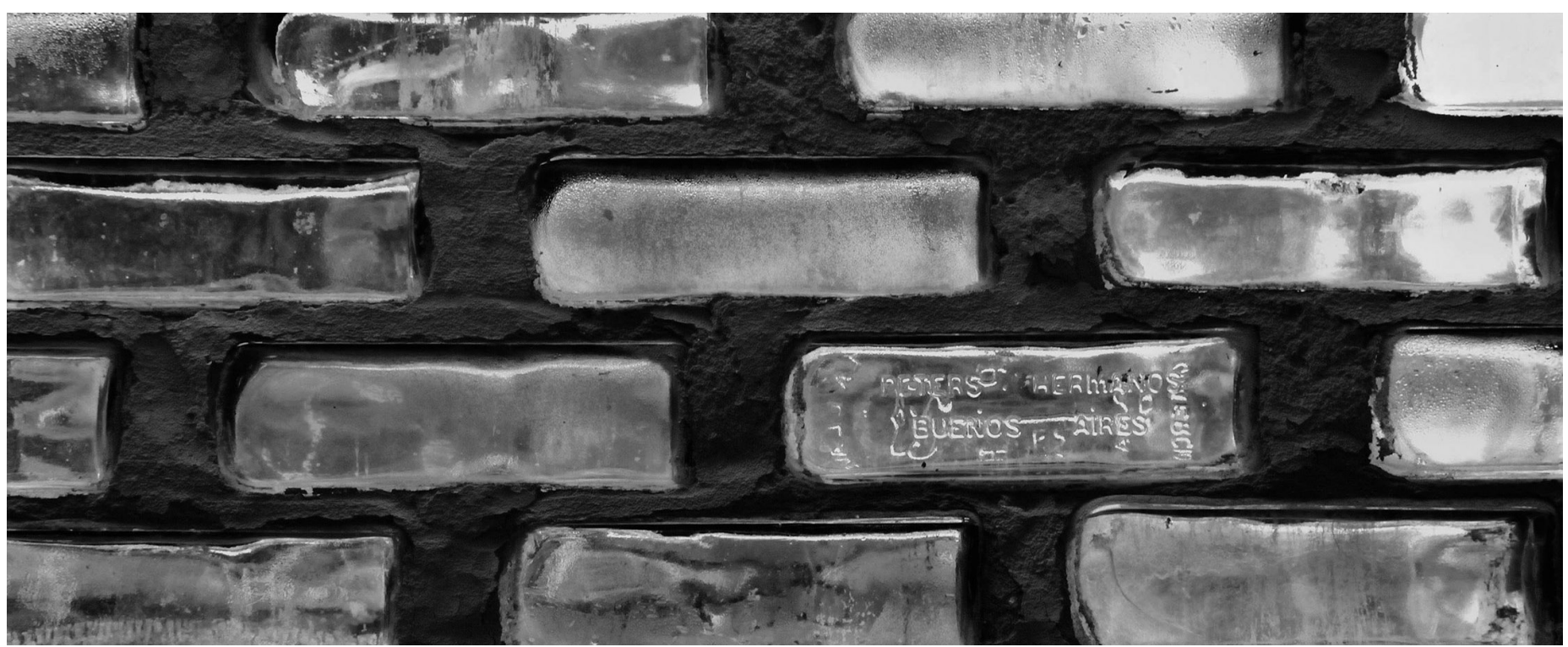

(c) Oscar Dechiara

Como se inferirá, la intervención formativa relacionada a esta dimensión simbólica no lo será tal sino se considera de manera integral su interrelación con las otras dimensiones. Esa es nuestra experiencia pedagógica que supone acompañar las trayectorias empíricas en el marco de los procesos políticos que le son inherentes, a partir de un abordaje eminentemente cultural. De tal manera, es indispensable considerar en nuestra práctica docente la concepción de territorio a la cual adscribimos, lo que nos permitirá abordar las trayectorias empíricas en su anclaje político y cultural, "evitando aislar artificialmente a los actores sociales de su contexto de acción e interrelación" (Pastore y Altschuler, 2015:116). Desde los aportes de la geografía social crítica de fines de 1970, podemos afirmar que no consideramos al territorio como un espacio determinado e inmutable, en una continuidad entre territorios, tan solo en su relación omnipresente con los poderes involucrados o en su apelación a su devenir como constructo del Estado-nación, sino al territorio como algo fijo o inmutable, como un espacio múltiple con configuraciones históricas flexibles y cambiantes (Pastore y Altschuler, 2015). Se trata de territorios superpuestos, discontinuos y en red, que dan cuenta de una estructura económica y social, atravesamientos de fuerzas y relaciones de poder de acuerdo con las instituciones que comandan la vida local y las restricciones impuestas desde las escalas provinciales y nacionales y también dinámicas de cambio (Altschuler, 2013). Como lo indican Altschuler y Pastore (2015:16), debe considerarse al

"territorio como un sujeto activo, entendido no en su sentido topográfico sino en su complejidad, incluyendo su historia, sus recursos naturales, económicos, culturales y sociales, poniendo de relieve el modo de relacionamiento" (16).

\section{Una tecnicatura universitaria en ESS}

Describiremos esta experiencia formativa a partir de nuestras observaciones y reflexiones como parte del equipo docente de las PP en la TUESS de la UNQ, desde que se creara la carrera en el año 2011. La TUESS se constituye como una experiencia de curricularización de la extensión universitaria dado que su matrícula proviene del DOSESS: a quienes cursaron el DOSESS (trayecto de extensión) se le reconocen 6 materias de la TUESS y comienzan a cursar $2^{\circ}$ año.

Estos estudiantes son trabajadores/as de origen y han sido desocupados durante los años del neoliberalismo, desplegando la potencialidad del campo de la economía popular y social desde diversas experiencias. Muchos de ellos son emprendedores autogestivos o intentaron serlo, expertos en prácticas populares de supervivencia económica como changarines, vendedores ambulantes, revendedores de ropa, productores de panificados caseros, etc. Algunos son el fruto de experiencias más elaboradas de la ESS (mutuales, cooperativas, redes de comercialización). Una gran mayoría proviene de un acuerdo con el Programa de Inserción Social con Trabajo del Ministerio de Desarrollo Social de la Nación. Son pobres estructurales o provienen de sectores empobrecidos, promovidos a partir de las políticas públicas para organizarse en cooperativas de trabajo en el territorio y estudiar en la Universidad. Muchos son militantes de organizaciones sociales y agrupaciones partidarias, en fuerte conexión con los entramados políticos locales y con conocimiento de las prácticas clientelares en sus distritos; algunos son actualmente técnicos de programas públicos vinculados a la ESS y al trabajo social comunitario. Tomando la experiencia desde el punto de vista etario y territorial, la diversidad del estudiantado es muy amplia: desde los 20 


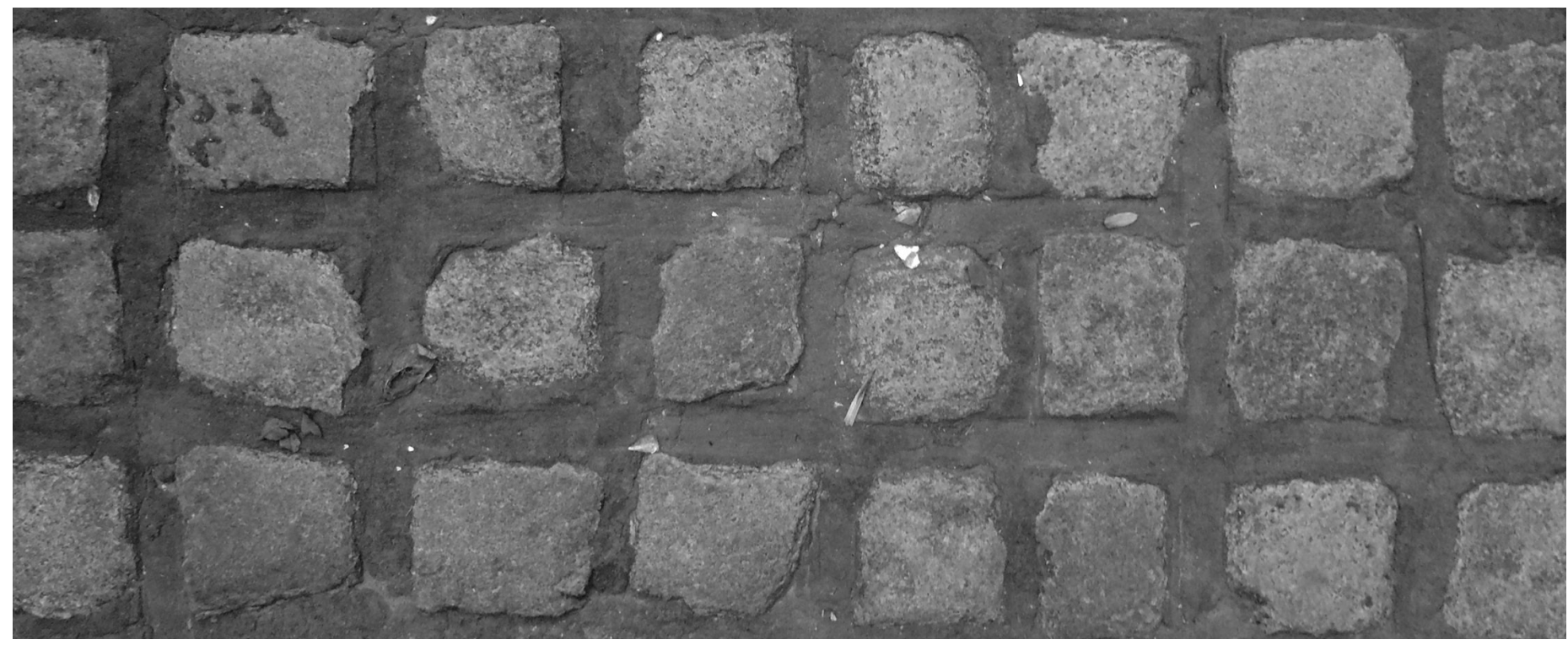

(C) Hugo Pascucci

años a los 60, viven en zonas urbanas y periurbanas del Área Metropolitana extendida de Buenos Aires, en una superficie que abarca un radio de $80 \mathrm{~km}$ alrededor de la ciudad de Buenos Aires, de manera tal que las prácticas territoriales donde se insertan también adquieren una notoria variabilidad y riqueza. Dirigida a este universo poblacional diverso, la TUESS se ha formalizado como la primera oferta de tecnicatura universitaria en la temática en Argentina y propone Prácticas Profesionalizantes (PP) pensadas como espacios de articulación interactoral (estudiantes-actores de la ESS) e intersectorial (sector de la ESS-sectores de la economía tradicional-Sector Público) con el objeto de formar dirigentes de la ESS.

Concebir las PP en ESS como contribuciones al desarrollo de las dimensiones empíricas, políticas y simbólicas que la constituyen nos desafía a construir e instaurar un novedoso campo profesional que al interior de las prácticas universitarias ha implicado fortalecer el vínculo de los proyectos de PP con la extensión, en tanto ésta participa de procesos de organización territorial y promueve la responsabilidad social y abona a la consideración de esta dimensión como un objetivo vertebral para la educación superior y una consecuencia lógica respecto de la búsqueda de la calidad educativa. El concepto de calidad educativa se enriquece con la significatividad y pertinencia de los contenidos en cuanto a las necesidades concretas de los estudiantes, proponiéndose más bien una creación de sentidos políticos y culturales que cualifican la práctica educativa (Dahlberg, Moss y Pence, 2006).

Dicha experiencia ha requerido la recreación de tecnologías didáctico-pedagógicas para dialogar con aquellos territorios móviles y cambiantes, con las prácticas en ellos desarrolladas y las vivencias de sus protagonistas: los estudiantes como sujetos de derecho y como trabajadores autogestionados, con historias personales y colectivas, con realidades plurales. Desde esa realidad se definen y articulan los proyectos de PP.

En este sentido, nos situamos desde la perspectiva de las Comunidades de Aprendizaje (Torres, 2013) en tanto proyectos educativos y culturales asumidos en los territorios desde sus mismos actores para ser llevados adelante desde la participación responsable y solidaria de todos y como dispositivos de formación de los sujetos adultos. Esta lógica de trabajo se propone en el aula y se transfiere a la práctica territorial. Es la modalidad pedagógica que orienta las prácticas, entendiendo la Comunidad de Aprendizaje ( $\mathrm{CdA}$ ) como un espacio que promueve el lazo social y la construcción compartida de saberes o, como plantea Torres, que integra los recorridos de la educación popular y la educación comunitaria latinoamericana, asumiéndola:

"no solo como un modelo local sino como una propuesta de política educativa; no resulta de la suma de intervenciones aisladas, o incluso de su articulación, sino que implica la construcción de planes educativos territorializados (el barrio, la comunidad, la cuadra, la manzana, el pueblo, la ciudad, el distrito, el municipio, etc.)". (Torres, 2013:56)

Los otros sujetos de la acción pedagógica, protagonistas desde el rol docente, provenimos de diversos campos disciplinares: somos sociólogos, psicólogos, politólogos, comunicadores sociales, trabajadores sociales, economistas, etc. Esta diversidad nos desafía a asumir la complejidad de la construcción interdisciplinar como el mejor aporte a la formación en ESS y es un aspecto fundamental en la conformación de este campo. 


\section{se procura resignificar las interacciones en las que se compromete la subjetividad, punto de articulación de procesos grupales, institucionales y colectivos, poniendo en discusión matrices ideológicas}

\section{Los Proyectos de las Practicas Profesionalisantes}

En el rol docente nos enfrentamos al desafío de atravesar las propias matrices de la formación académica tradicional donde las PP se asimilaban a instancias de "aplicación" de la teoría sobre la práctica, prácticas mayoritariamente realizadas en el trayecto final de la carrera, y supervisadas por otro docente con la misma formación y mayor experticia.

En nuestra Tecnicatura, las PP contrastan e integran los contenidos temáticos de las otras materias curriculares y el territorio, articulándose en Unidades Pedagógicas que se diseñan de manera conjunta entre docentes y estudiantes que son los Proyectos de PP. Éstos preforman la significatividad de los contenidos específicos del espacio curricular en función de las necesidades del desarrollo territorial y del empoderamiento y autonomía de los y las estudiantes en sus contextos, en tanto estos se constituyen como actores sociales específicos: Técnico/as Universitario/as en ESS. El técnico/a se desarrolla de manera singular en las habilidades ciudadanas y profesionales esperadas, las que demanda la ESS en su territorio.

El aula y el territorio se articulan en una praxis transformadora, en una relación dialéctica práctica-reflexión-vuelta a la práctica. De esta manera, los resultados del trabajo en el espacio territorial de prácticas son retomados en el espacio áulico para su reflexión y socialización y para organizar la vuelta al territorio con nuevas herramientas (Sena et al., 2015). Como instancia de formación, trabajamos con diferentes instrumentos para diagnosticar de manera participativa (relevamiento, análisis, sistematización), diseñar, implementar y evaluar proyectos de prácticas profesionalizantes que potencien la construcción del rol de Técnico Universitario en Economía Social y Solidaria y se inserten en proyectos o acciones marco presentes en el territorio.
En síntesis, retomando la categorización propuesta por Pastore y Altschuler (2015), el dispositivo de las prácticas profesionalizantes intenta:

a) En lo empírico, identificar actores y problemáticas del territorio y de la comunidad e indagar en ellas; adquirir destrezas y aptitudes necesarias para el diagnóstico, la formulación, ejecución y evaluación de proyectos en ESS; potenciar y difundir recursos de sujetos, organizaciones, grupos y comunidades, entre otras cuestiones.

b) En lo político, proponer proyectos transformadores que se integren a la construcción colectiva de la ESS en los territorios de pertenencia. Complejizar la configuración territorial en sus dimensiones institucionales y políticas.

c) En lo simbólico, construir un discurso poniendo en valor los recursos territoriales del campo, integrando criterios y valores de la ESS y visiones del desarrollo local y promover el trabajo en equipo para la apropiación y socialización del conocimiento.

En los últimos años, venimos también articulando los proyectos de PP con los ejes de trabajo propuestos desde los 13 proyectos de extensión universitaria (PEU) que conforman el Programa de Extensión Universitaria CREES ICOTEA (Construyendo Redes Emprendedoras en Economía Social y Solidaria-Integración Social, Comunidad de aprendizajes, Tecnologías para la inclusión social y Arte comunitario), posibilitando y potenciando de igual manera el trabajo de los estudiantes en su territorio específico, en articulación con las organizaciones existentes.

En este sentido, los proyectos de PP se han ido definiendo sobre diversos ejes: Formación en ESS, Organización de Nodos de Consumo y Experiencias de Comercialización de la ESS, 
Acompañamiento de la Gestión de Emprendimientos y Empresas Sociales, Implementación de estrategias comunicacionales de la ESS, Fortalecimiento de encadenamientos productivos vinculados al sector turístico, Formación en Género y ESS, Cultura Comunitaria y ESS, etc. Los mismos se instrumentan anualmente atravesando las fases del ciclo de proyectos, implican la intervención territorial concreta y la reflexión sobre el rol llevado adelante por el técnico en formación durante dicha intervención.

\section{4. "De los grupos a los equipos de trabajo} en Economía Social y Solidaria", un ejemplo

En nuestro recorrido, registramos con los estudiantes la necesidad de encarar procesos sistemáticos de reflexión teórico-práctica sobre las problemáticas inherentes a los procesos grupales que se movilizan en organizaciones, emprendimientos y otros agrupamientos territoriales de la ESS. Habitualmente, se presentan demandas de intervención sobre problemáticas vinculares que surgen en los emprendimientos. A partir de un PEU orientado al fortalecimiento de actores de la ESS desde acciones socioeducativas y formativas, se propone el Curso de Extensión Universitaria: "De los grupos a los equipos de trabajo en el campo de la Economía Social y Solidaria". Con una carga horaria de 32 horas y sin requisitos de estudios previos, tiene el objetivo de alojar, acompañar y contribuir a la comprensión de lo singularsubjetivo e intersubjetivo en las formaciones grupales de las organizaciones que constituyen el campo de la ESS, en diversos escenarios sociales. Se procura resignificar las interacciones en las que se compromete la subjetividad, punto de articulación de procesos grupales, institucionales y colectivos, poniendo en discusión matrices ideológicas y del contexto histórico político que favorecen o no las construcciones grupales.

Desde el punto de vista gnoseológico, socializamos los modos en que diferentes corrientes de pensamiento conciben la especificidad de lo grupal y el su contexto de surgimiento. Como CdA, se abordan temáticas como comunicación, dinámicas de interacción y covisión grupal, conflictos y estrategias de abordaje, construcción colectiva y trabajo colaborativo, cultura de la solidaridad, autogestión y gestión social, construcción de poder y construcción grupal, equipos de trabajo, roles, etc. En cada encuentro, se procura indagar sobre los saberes que integran los repertorios de los cursantes, especialmente los aprendidos por transferencia de prácticas culturales o a partir de sus experiencias, las matrices inconscientes, lo oculto por naturalización, inercia o por adaptación al medio. Se pretende visibilizar saberes fácticos, formas de pensar y también sentimientos aprendidos, valorizando en todas las instancias del Curso la interacción y articulación de estos elementos a través de la construcción de escenas dramáticas, canciones y otros productos imaginados colectivamente. Apelamos a la perspectiva de Fals Borda (2009), quien utiliza para definir este tipo de prácticas utiliza el adjetivo "sentipensante", extraído de la observación del modo de actuar del por él llamado "hombre icotea", genéricamente el pescador de la ribera del río Grande de La Magdalena (Colombia) que combina la razón y el amor, el cuerpo y el corazón para acompañar sus técnicas de pesca.

Cada encuentro es acompañado y coordinado por docentes y tutores, lo que posibilita que becarios y estudiantes de la TUESS (en el marco de sus PP), como responsables de la experiencia, se vean fortalecidos en el diagnóstico, ya que aportan sus conocimientos sobre experiencias territoriales y contactos de referencia en los territorios donde se implementa el Curso. Desarrollan habilidades de trabajo en equipo, coordinación y adecuación de la intervención según los distintos territorios y aptitudes para la convocatoria y difusión de la propuesta, para la planificación de los encuentros y el registro de los mismos con diferentes formatos y para la aplicación de herramientas que promuevan otro posicionamiento al interior de sus organizaciones de referencia, con mayor claridad en la toma de decisiones para accionar a favor de la consolidación de experiencias de ESS sustentables.

\section{Atravesamientos analíticos y territoriales de la formación y la extensión}

Como se mencionó, la TUESS ha permitido que el proyecto original del DOSESS (trayecto formativo de extensión) se curricularizara y fuera reconocido como instancia académica por resolución del Consejo Superior de la UNQ. Esto nos ha planteado desafíos didácticos que no son habituales en la Universidad para el acompañamiento directo de la trayectoria educativa y territorial de cada estudiante.

Luego, la organización curricular de las PP es, a nuestro entender, el exponente más acabado de la curricularización de la extensión. Las dimensiones intrínsecas de la ESS direccionan desde los territorios la organización de los contenidos formativos de las PP. Es coherente con ello que las Unidades Pedagógicas o Proyectos de Prácticas se vinculen directamente con las problemáticas que atraviesan a la ESS:

“a) las dificultades de índole económica y tecnológica; b) la escasa visibilidad, confluencia organizativa y representación colectiva de sus actores, y c) el limitado desarrollo de su identidad simbólica, en el marco hegemónico en los modos instituidos de vivir y entender lo económico y el poder como amenaza, control o dominio. A nuestro juicio, todo ello revaloriza la apuesta por generar espacios sistemáticos de formación que contribuyan a potenciar capacidades y aprendizajes en estas dimensiones". (Pastore, 2014:78)

Además, como aporte a la dimensión política de la ESS, estos proyectos de prácticas se articulan dialécticamente con la 


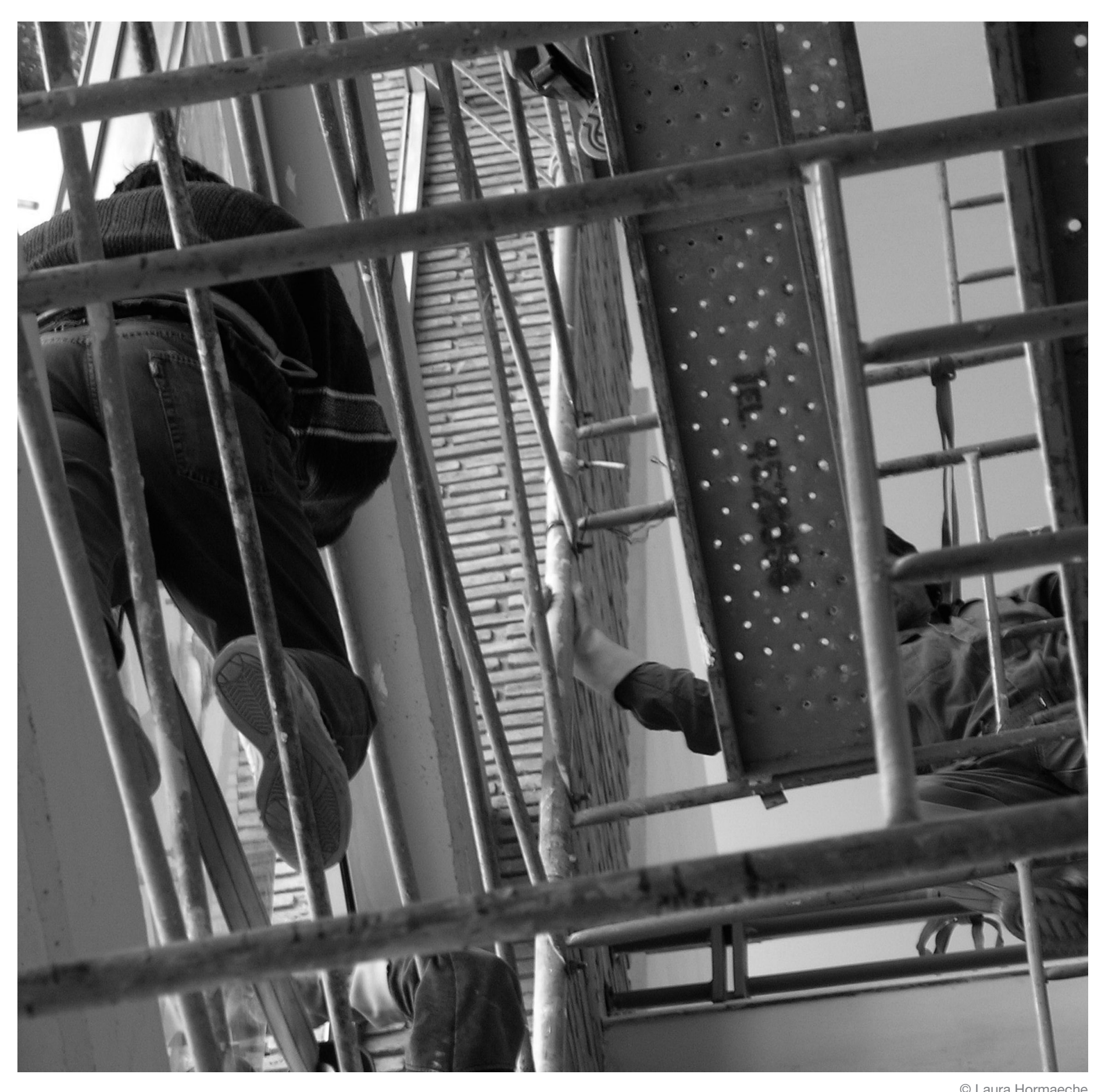


extensión y los territorios de incidencia de la ESS. La oferta formativa surge de la extensión, se integra a ella y vuelve hacia ella en tanto amplía y fortalece el campo de la ESS y el rol de la Universidad como actor de la red de la misma. Dicho de otra manera, el dispositivo pedagógico recupera las experiencias organizativas del territorio y diseña proyectos de prácticas que entraman la docencia y la extensión. Se provoca un tejido académico territorial que se hace texto y práctica en una espiral ascendente y profunda de transformaciones subjetivas y políticas. Todos los actores participantes somos desafiados a repensar las prácticas cotidianas en su potencialidad emancipatoria. Las PP retroalimentan esta sinergia y los egresados de la TUESS amplían territorios académicos y profesionales desde la extensión junto a sus organizaciones y en los territorios de referencia para que los actores colectivos de la ESS emerjan y se robustezcan.

Por otra parte, también comprobamos el aporte de las PP a la dimensión simbólica de la ESS que entrecruza las subjetividades y las culturas. Son nuestras subjetividades movilizadas en los recorridos de las prácticas, subjetividades bordeantes (Sena y Marciano, 2013), que atraviesan tanto a los docentes como a los estudiantes/militantes/técnicos en formación, dan respuestas nuevas a viejas prácticas y crean realidades nuevas a partir de la formación. Estas tensiones además cobran valor en función de ese sujeto de los bordes que ha emergido como estudiante, que provino de experiencias de trabajo y prácticas alejadas de las matrices de valoración habitual en la vida académica. En el aula circula dinámicamente desde él la complejidad de los territorios. Trabajar en los bordes, en los pasajes, en donde los quiebres en los que los saberes academizados entran en relación con los saberes de los territorios y viceversa nos ha ubicado desde el principio en una subjetividad afectada a partir de donde permanentemente vamos construyendo saberes entre todos, saberes que impactan en los territorios porque hablan de ellos o bien son los territorios los que hablan.

Las prácticas deben constituirse no como aplicación de contenidos externos a la cotidianeidad de nuestros estudiantes sino como una manera concreta de expresar las valoraciones de otra concepción educativa que rescata saberes, trayectorias subjetivas y se compromete con una construcción territorial concreta. Las PP, construidas desde y para el territorio, son dadoras de sentido y un eje que vertebra toda la propuesta de la Tecnicatura.

\section{Referencias bibliográficas}

Altschuler, B. (2013). Territorio y desarrollo: aportes de la geografía y otras disciplinas para repensarlos. Theomai, (27-28), 64-79.

Coraggio, J. L. (2011). Principios, instituciones y prácticas de la economía social y solidaria. Economía Social y Solidaria. El trabajo antes que el capital (pp. 345-405).

Quito: Ediciones Abya-Yala, FLACSO.

Coraggio, J. L. (2015). Desafíos en la formación profesional vinculados a la economía popular, las políticas públicas y el desarrollo local. El rol de la universidad. Revista $+E,(5), 6-19$.

Dahlberg, G.; Moss, P. y Pence, A. (2006). Más allá de la calidad en la educación infantil. Barcelona: Grao.

Fals Borda, O.; Moncayo, V.M. (Comp.) (2009). Una sociología sentipensante para América Latina. Bogotá: Siglo del Hombre Editores y CLACSO.

Pastore, R. (2014). Construyendo espacios universitarios de formación de actores de la Economía Social y Solidaria. Reflexiones desde una práctica académica-territorial. Voces en el Fénix, (38), 77-83.

Pastore, R. y Altschuler, B. (2015a). La economía social y solidaria, y los debates del desarrollo en clave territorial Reflexiones sobre experiencias y desafíos a partir de una práctica socioeducativa universitaria. Idelcoop, (217), 11-29.

Pastore, R. y Altschuler, B. (2015b). Economía social y solidaria en clave de desarrollo socio-territorial en Argentina. Conceptos, políticas públicas y experiencias desde la universidad. EUTOPIA, Revista de Desarrollo Económico Territorial, (7), 109-128. FLACSO Ecuador.

Sena, S. y Marciano, N.(2013) "La economía social y solidaria como constructora de una subjetividad bordeante". Interdisciplina e inclusión social como ejes de intervención. Buenos Aires: Asociación Argentina de Salud Mental.

Sena, S.; Sansón, D. y García, D. (2015). La Tecnicatura en Economía Social y Solidaria: Un espacio en construcción para estudiantes y docentes. VIII Jornadas Nacionales y I Congreso Internacional sobre la Formación del Profesorado. Facultad de Humanidades - Universidad Nacional de Mar del Plata; 29, 30 y 31 de octubre. Recuperado de: http://www.mdp.edu.ar/humanidades/pedagogia/jornadas/jprof2015/ponencias/sena.pdf

Torres, R.M. (2013). Comunidades de aprendizaje. En Valdés, R.; Pilz, D.; Rivero, J.; Machado, M.M.; Walder, G.; Fernández, C. y Capra, M. (2013). Aportes conceptuales de la educación de personas jóvenes y adultas: hacia la construcción de sentidos comunes en la diversidad. UNESCO para el Aprendizaje para toda la vida. Recuperado de: http://www.redetis.iipe.unesco.org/wp-content/ uploads/2015/10/aportes-conceptuales-de-la-Educacion-de-pers-Jovenes-yadultas.pdf (06/09/2016). http://www.fronesis.org/immagen/rmt/documentosrmt/ ComuApren4.pdf 